\title{
Microwave properties of HTS films: measurements in the millimeter wave range
}

\author{
N. T. Cherpak, ${ }^{\text {a) }}$ A. A. Barannik, Yu. V. Prokopenko, and Yu. F. Filipov \\ A. Usikov Institute of Radiophysics and Electronics of the National Academy of Sciences of Ukraine, \\ 12 Acad. Proskura Str., Kharkov 61085, Ukraine
}

\section{S. A. Vitusevich}

Institut für Schichten und Grenzfächen and Center of Nanoelectronic Systems for Information Technology, Forschungszentrum Jülich, 1 Leo-Brandt-Strasse, Jülich, 52425 Germany

(Submitted October 21, 2005; revised February 2, 2006)

Fiz. Nizk. Temp. 32, 795-801 (June 2006)

\begin{abstract}
A theoretical and experimental justification of an approach proposed and developed by us for surface impedance standard measurements of HTS films is presented. An analysis of the electromagnetic properties of quasi-optical dielectric resonators with conducting endplates, which provides a theoretical background for studies of HTS films in the millimeter wave range, is performed. With this technique, the highest-quality modes, namely whispering gallery modes, are excited in a dielectric cylindrical disc sandwiched between HTS films. Considerable enhancement of the sensitivity of surface resistance measurements in the millimeter wave range is demonstrated, which is important for the fundamental investigation of superconductor physics. It is also shown that the measured frequency shift in the resonator with the HTS endplates as a function of the temperature reveals a possibility for accurate evaluation of the field penetration depth in HTS films. (C) 2006 American Institute of Physics. [DOI: 10.1063/1.2215377]
\end{abstract}

\section{INTRODUCTION}

Microwave surface impedance $Z_{s}$ measurements of hightemperature superconducting (HTS) films are important for fundamental studies and also for technical applications. ${ }^{1}$ The resonant systems developed for contactless measurements of unpatterned films are of special interest. ${ }^{2}$ A well-known technique of surface resistance $R_{s}$ measurement based on a sapphire $\left(\mathrm{Al}_{2} \mathrm{O}_{3}\right)$ resonator with lower mode oscillations was used in the classic microwave band $(<25 \mathrm{GHz}){ }^{3}$ Various types of the Hakki-Coleman dielectric resonator have the advantage of being able to evaluate the total energy dissipation. ${ }^{4}$ The dimensions of dielectric resonators operating in the millimeter wavelength range $(>25 \mathrm{GHz})$ and utilizing lower-mode oscillations (waves) become unacceptably small. The quality factor $Q$ of the resonators decreases correspondingly. The highest quality factor can be obtained for the resonators using the highest order of azimuthal modes, i.e., whispering gallery (WG) waves [J. K. Wait, Radio Science 2, 1005 (1967)]. The dimensions of such a resonator in the millimeter wavelength range are acceptable for use in resonant structures with HTS films. ${ }^{5}$

We recently reported an accurate technique for the $R_{s}$ measurement of large-area HTS films using a sapphire quasioptical dielectric resonator (QDR) with conducting endplates (CEP). ${ }^{6}$ In this approach, the WG modes are excited in a dielectric cylindrical disc sandwiched between HTS films or between one HTS film and one copper endplate (Fig. 1). The approach was used in the Ka band and no fundamental limitations for use in a shorter wavelength range were found. It has been shown experimentally that the resonant frequency shift of QDR with HTS film as a function of the temperature can be used for accurate measurement of the surface reactance $X_{s}$ and, hence, a field penetration depth $\lambda=X_{s} /\left(\omega_{0} \mu_{0}\right)$, where $\omega_{0}=2 \pi f_{0} ; f_{0}$ is a resonant frequency, $\mu_{0}$ $=4 \pi \cdot 10^{-7} \mathrm{Hr} / \mathrm{m}$.

The aim of the present work is a complete analysis of the approach developed on the basis of QDR for HTS microwave surface impedance measurements which was reported briefly in Refs. 6, 8, and 9. The theoretical and experimental investigation both of the impedance properties of the HTS films and particular features of the proposed approach itself are highlighted here.

\section{ELECTRODYNAMICS OF QUASI-OPTICAL DIELECTRIC RESONATORS WITH CONDUCTING ENDPLATES}

The electromagnetic fields distribution of monochromatic oscillations in a disc QDR made of an anisotropic uniaxial single crystal with its anisotropy axis parallel to the disc axis is described in Ref. 10.

The frequency $\omega_{p}=\omega_{p}^{\prime}-i \omega_{p}^{\prime \prime}$ for the $p$ th mode of the resonant oscillation (where the subscript $p$ equals $n s m$, which are the azimuthal $(n)$, radial $(s)$ and axial $(m)$ subscripts, respectively) is found from a solution to the characteristic equation

$$
\left(\alpha_{H}-\alpha_{0}\right)\left(\varepsilon_{z} \alpha_{E}-\alpha_{0}\right)=a^{2},
$$

where

$$
\begin{aligned}
\alpha_{j} & =\frac{1}{q_{j} r_{0}} \frac{J_{n}^{\prime}\left(q_{j} r_{0}\right)}{J_{n}\left(q_{j} r_{0}\right)}, \quad \alpha_{0}=\frac{1}{q_{0} r_{0}} \frac{H_{n}^{(1)}\left(q_{0} r_{0}\right)}{H_{n}^{(1)}\left(q_{0} r_{0}\right)}, \quad a \\
& =\frac{k k_{z} n}{q_{0}^{2} q_{H}^{2} r_{0}^{2}}\left(1-\varepsilon_{\perp}\right),
\end{aligned}
$$

a prime denotes differentiation with respect to the argument; and $J_{n}(x)$ and $H_{n}^{1}(x)$ are the $n$ th-order Bessel and Hankel functions of the zeroth kind. For resonators with perfectly conducting endplates, the axial component of the wave vector is $k z=m \pi / l$, where $l$ is the height of the resonator and 


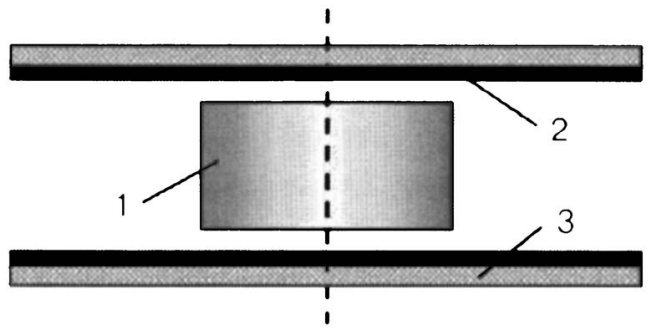

FIG. 1. Quasi-optical dielectric resonator with conducting endplates: 1-sapphire disc; 2-HTS films; 3-dielectric substrates.

$m=0,1,2, \ldots$ The subscript $j$ stands for $H$ or $E ; k=\omega / c$; the azimuthal subscript $n$ equals $0,1,2, \ldots ; q_{H}=\left(\varepsilon_{\perp} k^{2}-k_{z}^{2}\right)^{1 / 2}$; $q_{E}=\left(\varepsilon_{z} / \varepsilon_{\perp}\right)^{1 / 2} q_{H}$ and $q_{0}=\left(k^{2}-k_{z}^{2}\right)^{1 / 2}$ are the radial components of the wave vector inside and outside the insulator, respectively; $C_{n}$ and $D_{n}$ are constant factors; and $q_{2}=q_{H}^{2}$ when $r \leq r_{0}$ and $q^{2}=q_{0}^{2}$ when $r \geq r_{0}$.

A QDR supports independent EH and HE oscillations. When the condition

$$
\left(\alpha_{H}-\alpha_{0}\right)^{1 / 2}\left(\varepsilon_{z} \alpha_{E}-\alpha_{0}\right)^{-1 / 2} \gg 1
$$

is met, the free oscillations of the resonator are of the $\mathrm{HE}$ type.

If the axis of the uniaxial crystal forms an angle $\theta$ with the geometrical axis of the resonator, the permittivity tensor components depend on the azimuthal angle $\varphi$. This dependence splits the $\mathrm{EH}$ and $\mathrm{HE}$ oscillations into the ordinary and extraordinary modes, as follows from experiments. ${ }^{11}$

For the case where the endplates are not perfectly conducting planes, a rigorous theory does not exist. In the present work, the radial and azimuthal components of the wave vector are described by the characteristic equation (1), and the effective height of the resonator is defined as $l_{\text {eff }}$ $=m \pi / k z$.

The quadrature relations obtained from Maxwell's equations for resonant oscillations in resonators with perfectly and imperfectly conducting endplates is integrated over the entire space. ${ }^{12}$ Using the radiation condition, the continuity of the tangential electric and magnetic field components at the curved surface of the resonator, and the impedance boundary condition at the end faces of the resonator, one can obtain an integral equation that defines a shift in the resonance frequency due to imperfect conductivity of the CEP:

$$
\left(\omega-\omega_{p}^{*}\right)\left(\mathbf{H} \mathbf{H}_{p}^{*}+\mathbf{E}\left[\varepsilon_{i j}\right] \mathbf{E}_{p}^{*}\right) d V=
$$

V

$$
=2 i Z_{s}\left[\mathbf{e}_{z}\left[\mathbf{e}_{z}, \mathbf{H}\right]\right] \mathbf{H}_{p}^{*} d S
$$

$$
\text { S }
$$

where $\mathbf{e}_{z}$ is a unit vector along the QDR axis; $\omega, \mathbf{E}$, and $\mathbf{H}$ are the frequency and the electric and magnetic field vectors for the resonator with the imperfectly conducting endplates; and $\omega_{p}, \mathbf{E}_{p}$, and $\mathbf{H}_{p}$ are the frequency and the electric and magnetic field vectors for the $p$ th mode of the resonator with the perfectly conducting endplates. On the left-hand side of this formula, integration is performed over the entire volume
$V$; and on the right-hand side, over the surface $S$ of the CEP having the surface impedance $Z_{s}=R_{s}+i X_{s}$.

When $Z_{s}$ is small and the mode interaction is neglected $\left(I_{p^{\prime} p}=0\right.$ if $\left.p^{\prime} \neq p\right)$, one can obtain a relationship

$$
\left(\omega-\omega_{p}^{*}\right)+i Z_{s} \frac{I_{p p}^{2}}{W_{p}}=0
$$

where $I_{p^{\prime} p}$ is the surface current induced on the finiteconductivity endplates by the electromagnetic wave, and $W_{p}$ is the energy of the $p$ th electromagnetic mode in the resonator with the perfectly conducting endplate.

Relationship (5) can be used to calculate the surface impedance $Z_{s}$ of the CEP from the measured QDR resonance frequency $\omega$. We also use (5) for evaluation of the expected frequency shift of the QDR with HTS CEP. This relationship defines the shift between the resonance frequencies of a QDR with the imperfect CEP and that with the perfect CEP for a particular oscillation mode. The shift in the real part of the resonance frequency is specified by the imaginary part of the surface impedance $Z_{s}$ (the surface reactance $X_{s}$ ). The reactance specifies the nondissipative energy stored in the surface layers of the CEP. The surface resistance $R_{S}$ is responsible for the period-averaged Joule loss $I_{p^{\prime} p}^{2} R_{s}$ in the impedance CEP of the resonator.

The evaluations show that resonant frequencies of the QDR made of Teflon with copper CEP differ from those for the resonator with perfect CEP by $\sim 10 \mathrm{MHz}$. The estimated QDR frequency shift at superconducting-to-normal state transition in HTS CEP is approximately equal to the same value. This shift ensures a sufficient sensitivity for reactance measurements of HTS films.

The surface resistance $R_{s}$ of the CEP is more convenient to determine using a direct definition of the quality factor $Q$ in terms of the stored and dissipative energies:

$$
Q_{0}^{-1}=k \tan \delta+A_{s} R_{s}+Q_{\mathrm{rad}}^{-1},
$$

where $Q_{0}$ is the eigen $Q$-factor of QDR with CEP, coefficients $k$ and $A_{s}$ show the contribution of dielectric and conductor losses in the energy total loss in QDR, $\tan \delta$ is the dielectric loss tangent, and $Q_{\text {rad }}$ is the radiation loss quality. As was shown in Ref. 6 one can neglect $Q_{\text {rad }}^{-1}$ in QDR in the case of CEP with a large diameter $\left(Q_{\mathrm{rad}}=10^{9}-10^{10}\right)$.

The expressions for $k$ and $A_{s}$ can be obtained from the electrodynamic solution of the field structure in the QDR with $\mathrm{CEP}^{10}$

$$
k=1 /\left(1+R_{0}^{Y}\right)
$$

$$
A_{s}=2 /\left(\omega_{0} \mu_{0} l R_{Y}\right) \text {. }
$$

The expressions for $R_{Y}^{0}$ and $R_{Y}$ (index $Y$ means $\mathrm{HE}$ or $\mathrm{EH}$ mode) depend on the dimensions of the resonator and the wave mode within it. ${ }^{13}$ In the case of the axially homogeneous $\mathrm{HE}_{n s 0}$ mode, which is excited relatively easily, expressions for $R_{Y}^{0}$ and $R_{Y}$ are cited in Ref. 6. The coefficient $k$ is very close to 1 and the coefficient $A_{s}$ is shown in Fig. 2 as a function of the azimuthal index $n$ together with the corresponding values of frequencies for the $\mathrm{HE}_{n 10}$ and $\mathrm{HE}_{n 20}$ modes. It can be underlined that (i) $A_{s}$ decreases with increasing frequency, and (ii) the values of $A_{s}$ are the same for 


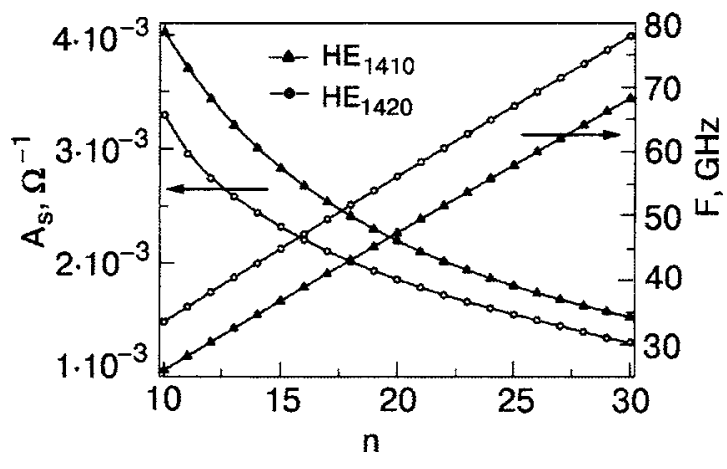

FIG. 2. The coefficient $A_{s}$ and resonant frequency dependence on azimuthal index $n$ for $\mathrm{HE}_{n 10}$ and $\mathrm{HE}_{n 20}$ modes. Symbols correspond to calculated values at the corresponding azimuthal index $n$.

the $\mathrm{HE}_{n 10}$ and $\mathrm{HE}_{n 20}$ modes at the same frequency (here, however, the values of $n$ are different for different modes).

\section{THE QDR-BASED APPROACH TO SURFACE RESISTANCE MEASUREMENT}

The main element of the measurement setup is a sapphire $(l=2.41 \mathrm{~mm})$ or ruby $(l=2.54 \mathrm{~mm})$ cylindrical disc with diameter $d=14.40 \mathrm{~mm}$. The optical $c$ axes of the single crystals were found at the angles $56^{\prime}$ and $15^{\prime}$ with respect to the geometric (longitudinal) axis for sapphire and ruby, respectively. Values of the loss tangent $(\tan \delta)$ measured at $77 \mathrm{~K}$ were found to be $2.9 \times 10^{-6}$ and $2.3 \times 10^{-6}$, respectively. The coupling of the QDR to the transmission lines was formed by dielectric waveguides with one side covered by metal (quasi-image waveguides). The angle between the longitudinal axis of the input and the output waveguides was about $10^{\circ}$. This allowed us to control the coupling between the QDR and the feeder lines by moving the resonator between these lines.

A small splitting of the resonance line was observed at $R_{S}$ measurements of high-quality HTS films forming CEP (Fig. 3). The $Q$-factor and $R_{s}$ in the case of a split resonance can be determined also. However, in this case it is necessary to develop the special approach to determine the $Q$-factor. ${ }^{6,14}$ At the beginning, test measurements were carried out with annealed $\mathrm{Cu}$ which showed very good coincidence of the measured and calculated values of $R_{s}$ at $T=290 \mathrm{~K}(48.8 \mathrm{~m} \Omega$ and $48.4 \mathrm{~m} \Omega$, respectively). The experimental data on $R_{s}$ $\left(\mathrm{m} \Omega\right.$ ) of three $\mathrm{YBa}_{2} \mathrm{Cu}_{3} \mathrm{O}_{7-\delta}$ films, measured at $T=78 \mathrm{~K}$ us-

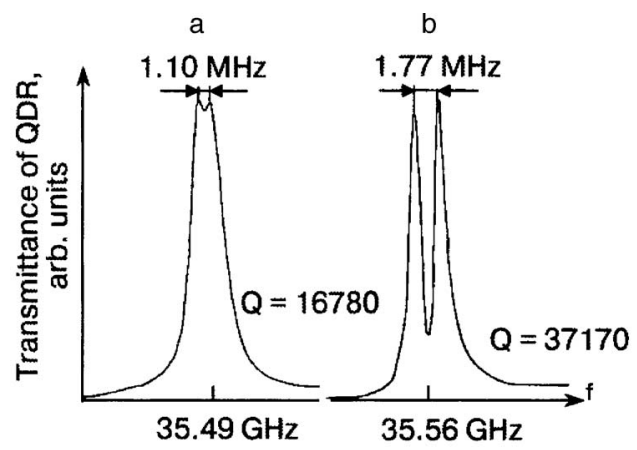

FIG. 3. Transmittance (i.e., resonance lines) of different QDR configurations in the Ka band at $T=77 \mathrm{~K}:(a)$ a sapphire disc with one HTS film and one $\mathrm{Cu}$ endplate; $(b)$ a sapphire disc with two HTS film endplates.

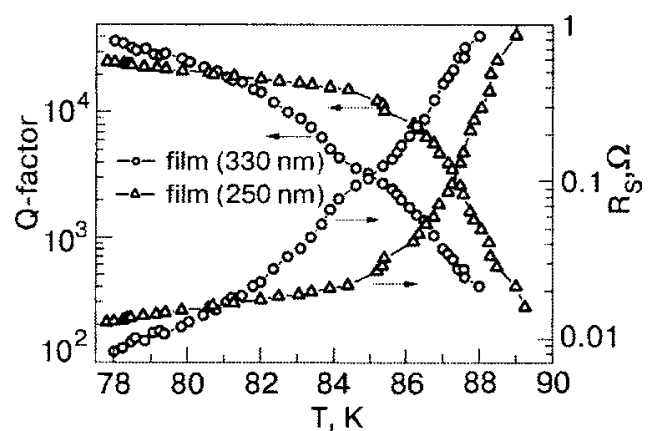

FIG. 4. Temperature dependence of a quality factor and surface resistance of a quasi-optical dielectric resonator with HTS films.

ing a "round-robin" procedure, ${ }^{15}$ are as follows: film $1-9.7$, film 2-8.3, film 3-10.1. As an example Fig. 4 shows the temperature dependence of $R_{s}$ for two different $\mathrm{YBaCuO}$ films.

For accurate $R_{s}$ measurements both a dielectric with minimal loss $\tan \delta$ and the oscillation wave mode with maximal $A_{s}$ should be used. The coefficient $R_{H E}$ depends slightly on the resonator geometric dimensions, the resonator material permittivity $\varepsilon^{\prime}$, and the wave mode. For the sapphire resonator one obtains $A_{s}=2.92 \times 10^{-3} \Omega^{-1}$ and $\delta A_{s} / A_{s}$ $\approx 0.5 \%$. It should be noted that $\delta R_{s} / R_{s}$ depends on the value of $R_{s}$ itself. Using the approach discussed in Ref. 3, the effects of other several factors on $\delta R_{s} / R_{s}$ have been analyzed in Ref. 16. The accuracy of the $R_{s}$ measurements is $5.5 \%$ under the conditions of the given case, i.e., $\tan \delta=2.3$ $\times 10^{-6}, \delta(\tan \delta) / \tan \delta=5 \%, \delta k / k=0.5 \%$, and can be improved to $1.5 \%$ by improving the $Q$-factor measurement accuracy to $1 \%$.

Using the above-mentioned approach, ${ }^{3,16}$ the minimum value of $R_{s}$ available for measurement can also be calculated:

$$
R_{s}^{\min }=\left|\frac{\delta Q_{0}}{Q_{0}}\right| \frac{k \tan \delta}{A_{s}} .
$$

As follows from (9), $R_{s}^{\min }$ is determined by the resolution of the $Q$-meter, loss tangent of the dielectric, and the filling factor $A_{s}$ of the superconductor. It can be seen that even at $\delta Q_{0} / Q_{0}=0.1$ and $\tan \delta=10^{-6}$, the value of $R_{s}^{\min }$ is equal to $4 \times 10^{-5}$. This value is lower than $R_{s}$ for the best HTS films in the Ka band, ${ }^{1}$ which allows one to perform new experiments on fundamental study of $R_{s}$ at very low temperatures.

As a result, the QDR-based technique of $R_{s}$ measurement of HTS films has a number of distinguishing features, namely:

- the technique is convenient for application in the millimeter wavelength range;

- the technique does not demand a calibration procedure, i.e., it is a first principles measurement technique;

- for QDR with CEP, axially homogeneous WG modes $\mathrm{HE}_{n s 0}$ are excited the most efficiently (where $n$ is the azimuthal index, $s$ means the number of field variations along the radius of the dielectric disc, and 0 means a uniform field distribution along the disc height);

- at excitation of the resonator in a regime of traveling waves, an observed resonant line splits, as a rule; 
- there is the opportunity to apply distributed and controlled (in a same temperature cycle of measurement) coupling with transmission lines.

The above mentioned features allow us:

- to achieve the highest sensitivity of surface resistance measurement at liquid helium temperatures, which opens up a new approach for studying the problem of microwave residual resistance both in HTS materials and other unusual superconductors;

- to measure microwave properties of large-area HTS films;

— to develop submillimeter $(\mathrm{THz})$ technique for impedance measurements;

- to consider QDR with CEP as the basis for developing of new microwave devices in millimeter-wave range (for example, high-stability oscillators, filters, etc.).

\section{APPROACH TO SURFACE REACTANCE STUDY}

Surface reactance $X_{s}$ is the next important impedance characteristic of the HTS thin film. As a rule, the same resonant structure is used for both $R_{s}$ and $X_{s}$ measurements. However, this method does not allow one to obtain absolute values of $X_{s}$ (in contrast to $R_{s}$ ), which is due to the impossibility of determining the eigenfrequency of resonators with ideal conducting surfaces and the insufficient reproducibility of the frequencies upon reassembling the resonator.

Consideration of the above-mentioned difficulties allows one to conclude that, evidently, in a given case, analogously to all other resonator techniques, the most appropriate approach can be one in which the reactance variation $X_{s}(T)$ with temperature is determined and the relations ${ }^{1}$

$$
\begin{aligned}
& \Delta X_{s}(T)=-\frac{2 \Delta f}{A_{s} f_{0}} ; \\
& \Delta \lambda(T)=\frac{\Delta X_{s}(T)}{2 \pi f_{0} \mu_{0}}
\end{aligned}
$$

are used, where $\Delta f_{0}$ is the shift of the eigenfrequency and $\Delta \lambda(T)$ is the temperature variation of $\lambda$.

Here small air-gaps (microslots) which exist between the HTS films used as CEP and the dielectric disc with WG modes are one of the important considerations affecting accuracy of impedance measurements. It is worth noting that the air-gap effect was negligible in microwave measurements of the dielectric properties of low-loss materials by the lower-mode dielectric resonator method. ${ }^{17}$ This problem was later considered numerically in connection with the use of the same lower-mode dielectric resonator for HTS film characterization. ${ }^{18}$ It was confirmed that the calculated influence of air gaps between the sapphire disc and CEP on the $Q$-factor can be accepted as insignificant. However, as the calculations showed, the same air gap reduced the resonant frequencies of the resonator rather noticeably. Naturally, the calculated data demand experimental verification, all the more in the given case, where the resonator is a quasi-optical element with WG modes, because a rigorous solution of the electrodynamic problem of QDR with air-gap has not yet been obtained. In addition, it is important to determine the temperature dependence of the air-gap effect. We are forced
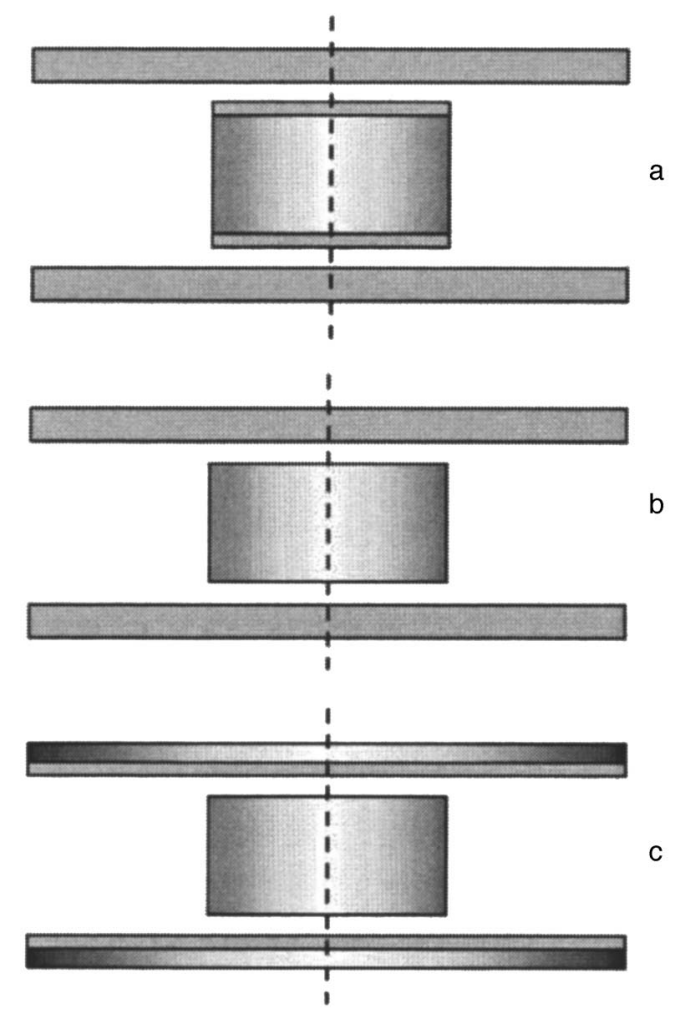

FIG. 5. Three versions of a quasi-optical dielectric resonator with conducting endplates in the form of copper films sputtered directly on dielectric disc $(a)$, copper bulk discs $(b)$, and copper films sputtered directly on dielectric substrates $(c)$.

to study this effect in QDR because it is distinct in eigenfrequency measurements upon reassembling of QDR with CEP.

To clarify the air-gap effect in QDR with CEP for $Z_{s}$ measurement, the structures shown in Fig. 5 were studied. In a structure shown in Fig. 5a, a dielectric disc of $14.40 \mathrm{~mm}$ in diameter with copper layers sputtered directly onto its end face is placed between bulk copper discs of $30 \mathrm{~mm}$ in diameter. The thickness of the copper layers is about $2 \mu \mathrm{m}$, i.e., $t \gg \delta \approx 0.35 \mu \mathrm{m}$, where $\delta$ is a skin depth. The copper discs are necessary to avoid the edge effect.

Comparison of resonant frequencies of the $\mathrm{HE}_{1410}$ mode for the structure shown in Fig. 5a (the air gaps are absent) and the structure shown in Fig. 5b (the air gaps are present) demonstrates that the air gap cause a frequency increase of $120 \mathrm{MHz}$. The use of springs to force the CEP onto the dielectric disc causes an increase of the frequency $\sim 300 \mathrm{MHz}$ (Fig. 5c). The latter is analogous to the case of QDR with HTS CEP.

However, despite the variation of the absolute value of the frequency, the temperature dependence of the frequency shift for structures in Figs. 5a and 5c is practically identical (Fig. 6). Therefore, the presence of the air gaps does not have a noticeable effect on the temperature dependence of the frequency shift $\Delta f$ which is used for the determination of $\Delta X_{s}(T)$ in accordance with (10a). The effect of the air gaps on $R_{s}$ measurement can be evaluated easily by using the coefficient $A_{s}$ dependence on frequency. This makes the error of the measured $R_{S}$ less than $1.5 \%$. Test $R_{S}$ measurements of the annealed $\mathrm{Cu}$ samples verify this conclusion. ${ }^{6}$ 


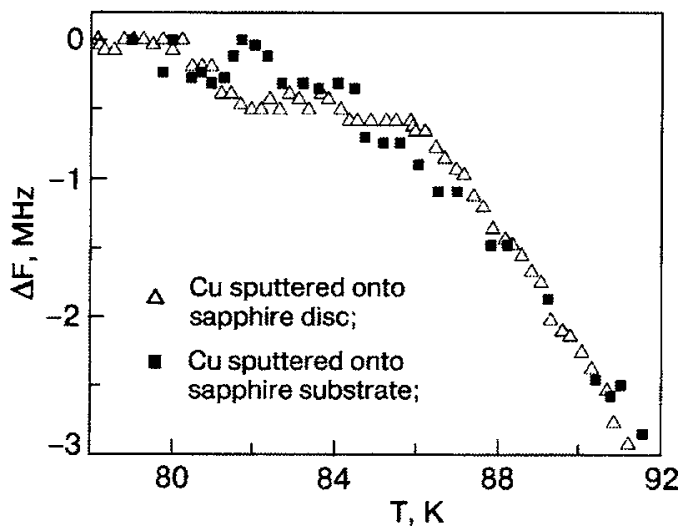

FIG. 6. Temperature dependence of resonant frequency shift of a quasioptical dielectric resonator with copper endplates.

In our case the dependence $X_{s}(T)$ for HTS films can be determined by using experimental dependence of the resonant frequency variation $\Delta f_{0}(T)$ with temperature for QDR with HTS films as CEP. However, the observed dependence $\Delta f_{0}(T)$ is related to both the variation of the reactance $X_{s}(T)$ and variation $\Delta \varepsilon(T)$ of the permittivity $\Delta \varepsilon(T)=\Delta \varepsilon^{\prime}(T)$ $+i \Delta \varepsilon^{\prime \prime}(T)$ of the dielectric of which the resonator is made, i.e.,

$$
\Delta f_{0 S}(T)=\Delta f_{S}(T)+\Delta f_{\varepsilon}(T) .
$$

Correctness of (11) follows from the condition $\Delta f_{0 S}(T) \ll \Delta f_{0}$. One can exclude $\Delta f_{\varepsilon}(T)$ from (11) by carrying out additional measurements $\Delta f_{0 N}(T)$ of QDR with CEP made of metal with the normal skin-effect

$$
\Delta f_{0 N}(T)=\Delta f_{N}(T)+\Delta f_{\varepsilon}(T) \text {. }
$$

From (11) and (12), we obtain the expression

$$
\Delta f_{0 S}(T)-\Delta f_{0 N}(T)=\Delta f_{S}(T)-\Delta f_{N}(T)=\Delta F(T) .
$$

For small variations $\Delta f_{\varepsilon}(T)$ the true relation is

$$
\Delta X_{s}(T)-\Delta X_{s}^{N}(T)=-\frac{2 \Delta F(T)}{A_{s} f_{0}} .
$$

In (14) an unknown $\Delta X_{s}^{N}(T)$ will remain. However, use of the normal metal allows one to determine $\Delta X_{s}^{N}(T)$ by its measured $\Delta R_{s}^{N}(T)$, because $\Delta X_{s}^{N}(T)=\Delta R_{s}^{N}(T)$. It is worth noting that measurements of frequency shift depending on tem-

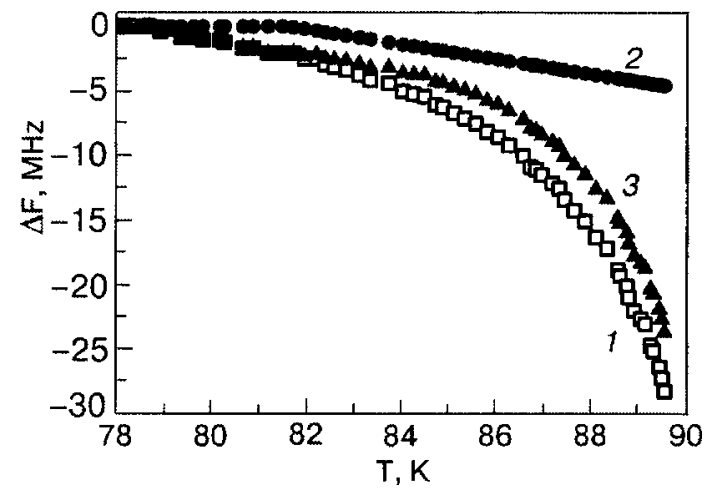

FIG. 7. Temperature dependence of resonance frequency variation for a quasi-optical dielectric resonator with conducting endplates: 1-Y-123; 2-Ti; 3-the difference of curves 1 and 2.

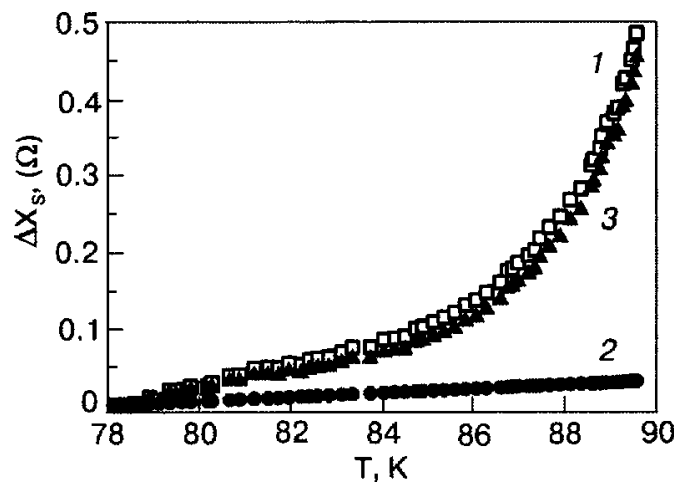

FIG. 8. Temperature dependence of surface reactance variation: $1-\mathrm{Y}-123$; 2-Ti; 3-the difference of curves 1 and 2 .

perature are carried out at rather weak coupling. Here the coupling change is shown to produce a negligible effect in the frequency shift, contrary to the change in a loaded quality factor. The approach described above was used for finding $X_{s}(T)$ of Y-123 films, the $R_{s}(T)$ of which was measured in a preceding Section. As a normal metal, Ti was used $\left(R_{S}\right.$ $=203.1 \mathrm{~m} \Omega, T=78 \mathrm{~K}$, Ka band). Figure 7 shows the temperature dependence of $\Delta f_{0 S}(T), \Delta f_{0 N}(T)$, and their difference $\Delta F(T)$. The Y-123 thin film dependence $X_{s}(T)$ obtained from (14) is presented in Fig. 8. The error of $X_{s}(T)$ in the temperature interval from $78 \mathrm{~K}$ to $89 \mathrm{~K}$ changes from $20 \%$ to $2 \%$ (at the absolute value $\approx 8 \mathrm{~m} \Omega$ of $X_{s}$ measurement error). It is necessary to emphasize that the $X_{s}(T)$ displayed in Fig. 8 is the dependence of the variation of the effective reactance, because the film thickness $d_{f} \approx \lambda$ and the surface impedance is a function of $d_{f}$. A connection of the effective impedance $Z_{s}=Z_{s}\left(d_{f}\right)$ with the intrinsic value $Z_{s}\left(d_{f} \rightarrow \infty\right)$ $=Z_{s \infty}$ can be found on the basis of the impedance transformation rule in view of the substrate properties. ${ }^{1}$ This enables us to compare the experimental and calculated dependences of $X_{s}(T)$ taking into account the film thickness $d_{f}$ (Fig. 9).

Restricting the work so far to only phenomenological models for $X_{s}(T)$ calculation, one can generalize an expression for $\lambda(T)$ on the basis of Refs. 19-21 in the form

$$
\lambda(T) / \lambda(0)=\left[1-\left(T / T_{s}\right)^{\gamma}\right]^{-n},
$$

where $\lambda(0)$ and the coefficients $\gamma$ and $n$ are fitting parameters. The use of different physical models results in different

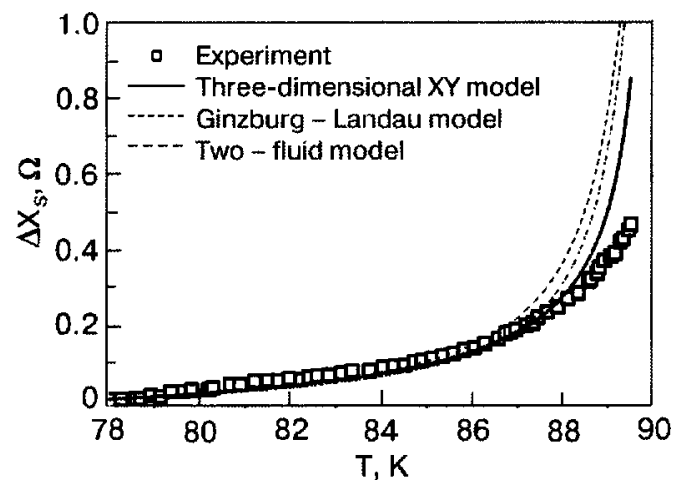

FIG. 9. Comparison of experimental and calculated dependences of the surface reactance of Y-123 thin films. 
TABLE I. Fitting parameters for three phenomenological models of $\lambda(T)$.

\begin{tabular}{c|c|c|c} 
Model & $\lambda(T), \mathrm{nm}$ & $n$ & $\gamma$ \\
\hline $\mathrm{XY}$ & 180 & $1 / 3$ & 1 \\
$\mathrm{GL}$ & 100 & $1 / 2$ & 1 \\
$\mathrm{TF}$ & 130 & $1 / 2$ & 2 \\
\hline
\end{tabular}

values of $\lambda(0), \gamma$, and $n$, which are obtained by applying a fitting procedure (Table I). As follows from Fig. 9 the XY (3D) critical regime approach follows the observed temperature dependence closer than Ginzburg-Landau (GL) behavior and the two-fluid (TF) model. This result seems to be in agreement with a number of measurements ${ }^{19}$ of the field penetration depth in Y-123 single crystals for temperatures very close to $T_{c}$ and is inconsistent with Ref. 21, where the experimental data have displayed good agreement with GL theory. However, it may also be appropriate to continue work on the accurate measurement and theoretical analysis of the temperature dependence of the surface reactance near $T_{c}$.

\section{CONCLUSION}

The QDR-based technique proposed and developed by us allows the investigation of HTS film microwave properties. It permits the highest sensitivity to be achieved in the millimeter wave range. The evaluation of the sensitivity enhancement obtained at very low temperatures indicates the possibility of studying residual microwave surface resistance, which is an important fundamental problem of hightemperature superconductivity. The technique is also useful for the study of reactance properties. No restrictions on developing this QDR-based approach to submillimeter $(\mathrm{THz})$ impedance measurements can now be found.
${ }^{a)}$ Electronic mail: cherpak@ire.kharkov.ua

${ }^{1}$ M. Hein, High-Temperature-Superconductor Thin Films at Microwave Frequencies, Vol. 155 of Springer Tracts in Modern Physics, BerlinHeidelberg-New York (1999).

${ }^{2}$ J. Ceremuga, J. Krupka, and T. Kosciuk, J. Supercond. 8, 681 (1995).

${ }^{3}$ J. Mazierska and C. Wilker, IEEE Trans. Appl. Supercond. 11, 3217 (2001).

${ }^{4}$ B. W. Hakki and P. D. Coleman, IEEE Trans. Microwave Theory Tech. 8, 402 (1960).

${ }^{5}$ N. T. Cherpak, MSMW'2001 Symposium Proc. (Kharkov) 1, 63 (2001).

${ }^{6}$ N. T. Cherpak, A. A. Barannik, Yu. F. Filipov, Yu. V. Prokopenko, and S. A. Vitusevich, IEEE Trans. Appl. Supercond. 33, 3570 (2003).

${ }^{7}$ N. T. Cherpak, A. A. Barannik, Yu. V. Prokopenko, and S. A. Vitusevich, Supercond. Sci. Technol. 17, 899 (2004).

${ }^{8}$ N. T. Cherpak, A. A. Barannik, Yu. V. Prokopenko, and S. A. Vitusevich, APMC'2004 Abstracts, New Delhi, India (2004), p. 1045.

${ }^{9}$ N. T. Cherpak, A. A. Barannik, Yu. F. Filipov, Yu. V. Prokopenko, and S. A. Vitusevich, IEEE Trans. Appl. Supercond. 15, 2919 (2005).

${ }^{10}$ Yu. V. Prokopenko and Yu. F. Filipov, Tech. Phys. 47, 731 (2002).

${ }^{11}$ A. Ya. Kirichenko, Yu. V. Prokopenko, Yu. F. Filipov, and N. T. Cherpak, Radiotekh. Elektron. (Moscow) 1, 300 (1989).

${ }^{12}$ Yu. V. Prokopenko and Yu. F. Filipov, Radiofiz. Elektron. (Kharkov) 6, 30 (2001).

${ }^{13}$ A. A. Barannik, Yu. V. Prokopenko, Yu. F. Filipov, and N. T. Cherpak, Telecommunications and Radioengineering 55, 360 (2001).

${ }^{14}$ A. A. Barannik, N. T. Cherpak, and D. E. Chuyko, IEEE Trans. Instrum. Meas. 55, 70 (2006).

${ }^{15}$ Zhi-Yuan Shen, High-Temperature Superconducting Microwave Circuits, Artech House, Boston-London (1994).

${ }^{16}$ A. A. Barannik, S. A. Bunyaev, and N. T. Cherpak, Radiofiz. Elektron. (Kharkov) 6, 201 (2001).

${ }^{17}$ Y. Kobayashi and M. Katoh, IEEE Trans. Microwave Theory Tech. 33, 586 (1985).

${ }^{18}$ J. Ceremuga, J. Krupka, R. Geyer, and J. Modelski, IEICE Trans. Electron. E78-C, 1106 (1995).

${ }^{19}$ S. M. Anlage, J. Mao, J. C. Booth, Dong Ho Wu, and J. L. Peng, Phys. Rev. B 53, 2792 (1996).

${ }^{20}$ S. Hensen, G. Muller, C. T. Rieck, and K. Scharnberg, Phys. Rev. B 56, 6237 (1997).

${ }^{21}$ A. Andreone, C. Cantoni, A. Cassinese, A. Di Chiara, and R. Vaglio, Phys. Rev. B 56, 7874 (1997). 